Research Article

\title{
Couple Stress Squeeze Films with VPD in a Curved Circular Geometry
}

\author{
Vimala Ponnuswamy $^{1}$ and Sumathi Govindaraj ${ }^{2}$ \\ ${ }^{1}$ Department of Mathematics, Anna University, Chennai 600025, Tamil Nadu, India \\ ${ }^{2}$ Department of Mathematics, Adhiparasakthi Engineering College, Melmaruvathur 603319, Tamil Nadu, India \\ Correspondence should be addressed to Vimala Ponnuswamy; vimalap@annauniv.edu
}

Received 12 July 2014; Revised 22 December 2014; Accepted 12 January 2015

Academic Editor: Ian Sherrington

Copyright (C) 2015 V. Ponnuswamy and S. Govindaraj. This is an open access article distributed under the Creative Commons Attribution License, which permits unrestricted use, distribution, and reproduction in any medium, provided the original work is properly cited.

\begin{abstract}
The problem of couple stress squeeze films considering viscosity pressure dependence (VPD) has been analysed in a curved circular geometry. Using Stokes microcontinuum theory and the Barus formula, the Reynolds type equation has been derived. The approximate analytical expressions for the squeeze film pressure and load carrying capacity are obtained using a perturbation technique. The numerical solutions for the squeeze film pressure and load carrying capacity are presented for the sinusoidal motion of the upper curved disk, assuming an exponential form for the curvature. The effects of curvature, the non-Newtonian couple stresses, and VPD and their combined effects are investigated through the squeeze film pressure and the load carrying capacity of the squeeze film.
\end{abstract}

\section{Introduction}

Many areas of Engineering and Science such as lubricated joints, gears, viscous dampers, bearings, and automotive and aircraft engines witness the major role of squeeze film phenomena. Squeeze film is a thin layer of viscous fluid between approaching surfaces. As the viscous lubricant is contained between the surfaces, it takes certain time for these surfaces to come into contact with each other and the lubricant cannot be squeezed out instantaneously. Since the viscous lubricant has a resistance to extrusion, a pressure is built up during this interval and then the load is supported by the lubricant film.

In most of the studies of classical hydrodynamic lubrication, it is assumed that the lubricant behaves as a Newtonian fluid as studied by Pinkus and Sternlicht [1] and Jones and Wilson [2]. In recent years, experimental results show that the addition of a small amount of long-chained polymers to a Newtonian fluid gives the most desirable lubricant. It has been experimentally showed by Spikes [3] that base oil blended with viscosity index improvers can enhance the behaviour of the lubricants in hydrodynamic contacts. Many microcontinuum theories have been developed by Ariman and Sylvester $[4,5]$ and Stokes [6] to describe the rheological behaviour of such non-Newtonian lubricants. Among these, the Stokes microcontinuum theory is considered as the simplest generalisation of the classical continuum theory of fluids, which allows the polar effects such as the presence of couple stresses and body couples. Several investigators such as Ramanaiah [7], Bujurke and Naduvinamani [8], and Alyaqout and Elsharkawy [9] considered Stokes microcontinuum theory for the study of various bearing systems because of its relative mathematical simplicity. In literature, investigations on the couple stress squeeze films between flat circular disks between a sphere and a flat plate and between porous surfaces have been carried out by Lin and Hung [10], Lin [11], and Naduvinamani et al. [12], respectively. However, the curved plate geometry is representative of many practical machineries involving hydrodynamic lubrication. It is observed that in a squeeze flow problem, the gap width between the approaching surfaces is small, when compared with the dimensions of the surfaces. Thus even if the order of waviness in the curved plates is small, the effects of nonflatness on the squeeze film flow is not necessarily negligible 
as shown by Gupta and Kapur [13], Usha and Vimala [14, 15]. Therefore, the effects of curvature on the squeeze film behaviour are also significant in this study.

The study of variation of viscosity with pressure on the isothermal, incompressible squeeze films between parallel disks by Butler [16] shows that the predicted fluid pressure is higher when VPD is taken into consideration than in the isoviscous case. The effects of variation of viscosity with pressure and temperature have been studied by Gould [17] and it has been shown that the above effects play a major role in squeeze film behaviour. Kalogirou et al. [18] have studied VPD in incompressible Poiseuille flow of Newtonian liquids. Lu and Lin [19] have studied the combined effects of couple stresses and VPD for the sphere-plate squeeze film system and observed that these effects enhance the load carrying capacity of the squeeze film.

The circular squeeze film system can be used in biolubricated joints such as synovial joints and clutch plates in automotive transmission. In this paper, a squeeze film flow of couple stress fluids between a flat circular fixed disk and a curved circular moving disk is considered taking VPD into account. Using Stokes microcontinuum theory and the Barus formula, the nonlinear Reynolds type equation is derived. The approximate analytical expressions for the squeeze film pressure and the load carrying capacity are obtained using a perturbation technique. Numerical results are obtained for the sinusoidal motion of the upper curved disk with the curvature described by an exponential form. The effects of couple stresses, curvature, and variation of viscosity with pressure and their combined effects on the squeeze film characteristics are investigated.

\section{Mathematical Formulation}

The unsteady, axi-symmetric laminar flow of an incompressible couple stress fluid between a flat circular lower disk at $z=0$ and a curved circular upper disk at $z=h(r, t)$ of radius $r_{a}$ is shown in Figure 1. The upper curved disk approaches the lower flat disk with a squeezing velocity $V_{s}=-h_{t}=-\partial h / \partial t$.

Assuming that the body forces and body couples are absent, the governing equations of motion of the incompressible couple stress fluid in the axi-symmetric case using Stokes microcontinuum theory take the form

$$
\begin{aligned}
\frac{1}{r} \frac{\partial(r u)}{\partial r}+\frac{\partial w}{\partial z} & =0 \\
\frac{\partial p}{\partial r} & =\frac{\partial}{\partial z}\left(\mu \frac{\partial u}{\partial z}\right)-\eta \frac{\partial^{4} u}{\partial z^{4}} \\
\frac{\partial p}{\partial z} & =0
\end{aligned}
$$

where $u$ and $w$ are the velocity components in $r$ and $z$ directions, respectively, $p$ is the film pressure, $\rho$ is the fluid density, $\mu$ is the shear viscosity, and $\eta$ represents the new material constant with the couple stress property. To consider

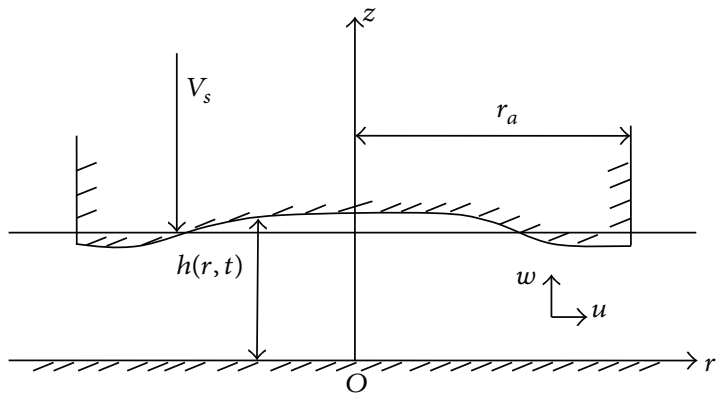

FIGURE 1: Curved squeeze film geometry.

the variation of viscosity with pressure, the Barus formula for isothermal VPD is

$$
\mu=\mu_{0} \exp (\alpha p)
$$

where $\mu_{0}$ is the viscosity at ambient pressure and at constant temperature and $\alpha$ is the pressure-viscosity coefficient. The boundary conditions for the velocity components are the noslip conditions given by

$$
\begin{aligned}
& u=0, \quad w=0, \quad \text { at } z=0, \\
& u=0, \quad w=-V_{s}, \quad \text { at } z=h(r, t),
\end{aligned}
$$

and the no-couple stress conditions given by

$$
\frac{\partial^{2} u}{\partial z^{2}}=0, \quad \text { at } z=0, \text { at } z=h(r, t) .
$$

The boundary conditions for the squeeze film pressure are

$$
\begin{gathered}
p=0, \quad \text { on } r=r_{a}, \\
\frac{\partial p}{\partial r}=0, \quad \text { at } r=0 .
\end{gathered}
$$

\section{Nondimensional Form of the Problem}

Introduce the following nondimensional quantities

$$
\begin{aligned}
R & =\frac{r}{r_{a}}, \\
Z & =\frac{z}{h_{0}}, \\
H & =\frac{h}{h_{0}}, \\
L & =\frac{l}{h_{0}}, \\
U & =\frac{h_{0} u}{r_{a} V_{s}}, \\
W & =\frac{w}{V_{s}} .
\end{aligned}
$$


Here $l=\sqrt{\eta / \mu}$ is the couple stress parameter of dimension length and this length may be regarded as the chain-length of polar additives in the lubricant. Using (2) and (6), the nondimensional form of the governing equations given by (1), take the form

$$
\begin{aligned}
\frac{1}{R} \frac{\partial(R U)}{\partial R}+\frac{\partial W}{\partial Z} & =0, \\
\frac{\partial P}{\partial R} & =\frac{\partial}{\partial Z}\left(\exp (\gamma P) \frac{\partial U}{\partial Z}\right)-L^{2} \frac{\partial^{4} U}{\partial Z^{4}}, \\
\frac{\partial P}{\partial Z} & =0,
\end{aligned}
$$

where the nondimensional form of squeeze film pressure $P$ is given by $P=p h_{0}^{3} / \mu r_{a}^{2} V_{s}$ and the viscosity parameter is $\gamma=\mu_{0} \alpha r_{a}^{2} V_{s} / h_{0}^{3}$. On nondimensionalization, the boundary conditions of the velocity components and pressure become

$$
\begin{aligned}
U & =0, \quad W=0, \quad \text { on } Z=0, \\
U & =0, \quad W=-1, \quad \text { on } Z=H(R, T), \\
\frac{\partial^{2} U}{\partial Z^{2}} & =0, \quad \text { on } Z=0 ; \\
\frac{\partial^{2} U}{\partial Z^{2}} & =0, \quad \text { on } Z=H(R, T), \\
P & =0, \quad \text { on } R=1 ; \\
\frac{\partial P}{\partial R} & =0, \quad \text { at } R=0 .
\end{aligned}
$$

Solving (8) with the boundary conditions (10) and (11), the radial velocity component is obtained as

$$
\begin{aligned}
U= & \frac{\exp (-2 \gamma P)}{2} \frac{\partial P}{\partial R} \\
& \cdot\left\{\frac{Z^{2}-H Z}{\exp (\gamma P)}+2 L^{2}-2 L^{2}\right. \\
& \left.\cdot \frac{\cosh [(2 Z-H) / 2 L \exp (-0.5 \gamma P)]}{\cosh (H / 2 L \exp (-0.5 \gamma P))}\right\} .
\end{aligned}
$$

Integrating the continuity equation (7) with respect to $Z$ and using the boundary conditions of axial velocity component from (10) and (11), the non-Newtonian Reynolds type equation is obtained as

$$
\frac{\partial}{\partial R}\left[f(H, L, \gamma, P) R \frac{\partial P}{\partial R}\right]=-12 R,
$$

where $f(H, L, \gamma, P)=H^{3} \exp (-\gamma P)-12 L^{2} H \exp (-2 \gamma P)+$ $24 L^{3} \exp (-2.5 \gamma P) \tanh (H \exp (0.5 \gamma P) / 2 L)$.

To find the approximate analytical solution for the highly nonlinear equation (14), the squeeze film pressure is expanded, using a small perturbation, in the form

$$
P=P_{0}+\gamma P_{1}+\gamma^{2} P_{2}+\cdots
$$

with $0 \leq \gamma \ll 1$. Substituting (15) in the Reynolds type equation (14) and in the pressure conditions the zero order problem becomes

$$
\frac{d}{d R}\left[f_{0}(H, L) R \frac{\partial P_{0}}{\partial R}\right]=-12 R,
$$

where $f_{0}(H, L)=H^{3}-12 L^{2} H+24 L^{3} \tanh (H / 2 L)$. The pressure conditions are given by

$$
\begin{gathered}
P_{0}=0, \quad \text { on } R=1 ; \\
\frac{\partial P_{0}}{\partial R}=0, \quad \text { at } R=0 .
\end{gathered}
$$

By solving (16) along with the boundary conditions given by (17), the zero order solution is obtained as

$$
P_{0}=6 \int_{R}^{1} \frac{R}{f_{0}} d R .
$$

Similarly, the first order problem becomes

$$
\frac{d}{d R}\left[f_{0}(H, L) \frac{\partial P_{1}}{\partial R}-f_{1}(H, L) P_{0} \frac{\partial P_{0}}{\partial R}\right]=0,
$$

where $f_{1}(H, L)=H^{3}-6 L^{2} H\left[4+\operatorname{sech}^{2}(H / 2 L)\right]+$ $60 L^{3} \tanh (H / 2 L)$ with

$$
\begin{gathered}
P_{1}=0, \quad \text { on } R=1 ; \\
\frac{\partial P_{1}}{\partial R}=0, \quad \text { at } R=0 .
\end{gathered}
$$

The first order solution is obtained as

$$
P_{1}=36 \gamma \int_{R}^{1} \frac{R_{2} f_{1}}{f_{0}^{2}} \int_{R_{2}}^{1} \frac{R_{1}}{f_{0}} d R_{1} d R_{2},
$$

where $R_{1}$ and $R_{2}$ are dummy variables of integration representing the radial coordinate $R$. Substituting $P_{0}$ and $P_{1}$ in (15), the approximate squeeze film pressure $P$ is obtained as

$$
P=6 \int_{R}^{1} \frac{R_{1}}{f_{0}} d R_{1}+36 \gamma \int_{R}^{1} \frac{R_{2} f_{1}}{f_{0}^{2}} \int_{R_{2}}^{1} \frac{R_{1}}{f_{0}} d R_{1} d R_{2} .
$$

The dimensionless load carrying capacity is now obtained by integrating the film pressure given in (22) as

$$
\begin{aligned}
F_{\mathrm{sq}}= & 2 \pi \int_{0}^{1} R P d R \\
= & 12 \pi \int_{0}^{1} R_{2} \int_{R_{2}}^{1} \frac{R_{1}}{f_{0}} d R_{1} d R_{2} \\
& +72 \pi \gamma \int_{0}^{1} R_{3} \int_{R_{3}}^{1} \frac{R_{2} f_{1}}{f_{0}^{2}} \int_{R_{2}}^{1} \frac{R_{1}}{f_{0}} d R_{1} d R_{2} d R_{3},
\end{aligned}
$$

where $R_{1}, R_{2}$, and $R_{3}$ are dummy variables of integration representing $R$ and the nondimensionalization for the load carrying capacity $f_{\mathrm{sq}}$ is given by $F_{\mathrm{sq}}=f_{\mathrm{sq}} h_{0}^{3} / \mu_{0} r_{a}^{4} V_{s}$. Equations (22) and (23) give the squeeze film pressure and the load carrying capacity for an arbitrary shape of the upper curved disk. 


\section{Solution for Sinusoidal Motion}

Assuming a specific form of $h(r, t)$ given by $h(r, t)=h_{1}(t) k(r)$ the problem is analysed. Here $h_{1}(t)$ represents the central film thickness and $k(r)$ is the curvature profile of the curved disk. The solution is obtained for a sinusoidal motion $h_{1}(t)=$ $h_{10}+e \sin \omega t$ of the upper curved disk. Here $h_{10}$ is the initial central film thickness and $e$ and $\omega$ are the amplitude and the angular frequency of the sinusoidal motion, respectively. Here the curvature profile is assumed to take an exponential form $k(r)=e^{-\widehat{c} r^{2}}$ where $\widehat{c}$ is the curvature parameter with $\widehat{c}>0$ for concave disk, $\widehat{c}<0$ for convex disk, and $\widehat{c}=0$ for a flat disk. Using the nondimensional quantities from (6) along with

$$
\begin{aligned}
H_{1} & =\frac{h_{1}}{h_{10}}, \\
E & =\frac{e}{h_{10}}, \\
T & =\omega t
\end{aligned}
$$

the dimensionless form of $h(r, t), h_{1}(t)$, and $k(r)$ is given by

$$
\begin{aligned}
H(R, T) & =H_{1}(T) k(R), \\
H_{1}(T) & =1+E \sin T, \\
k(R) & =e^{-c R^{2}},
\end{aligned}
$$

where $c=\widehat{c} r_{a}^{2}$. The configuration of the curved disk for various values of the curvature parameter $c$ is shown in Figure 2. The dimensionless squeeze film pressure and force can be obtained from (22) and (23) using (25). Considering the flat disk case, the gap-width becomes independent of the radial coordinate; that is, $h(r, t)=h(t)$. In this case, the squeeze film pressure and the load carrying capacity are obtained from (22) and (23) as

$$
\begin{aligned}
P & =\frac{3}{f_{0}}\left(1-R^{2}\right)+\frac{9 \gamma f_{1}}{f_{0}^{3}}\left[\frac{1}{2}-R^{2}\left(1-\frac{R^{2}}{2}\right)\right], \\
F_{\mathrm{sq}} & =\frac{3 \pi}{2 f_{0}}\left(1+\frac{\gamma f_{1}}{f_{0}^{2}}\right) .
\end{aligned}
$$

\section{Results and Discussions}

The combined effects of curvature, couple stresses, and variation of viscosity with pressure on the squeeze film behaviour are analysed using Stokes microcontinuum theory and Barus formula for VPD. The effects of couple stresses on the squeeze film characteristics are observed through the nondimensional couple stress parameter $L$, the curvature effects through the curvature parameter $c$, and the variation of viscosity with pressure through the viscosity parameter $\gamma$. Considering the sinusoidal motion of the upper curved disk, the squeeze film characteristics for the flow in the flat disk case are obtained, respectively, from (26) and (27). Here, a small amplitude $E=0.2$ is taken for the sinusoidal motion and time $T=0.8$ is chosen for a squeezing state.

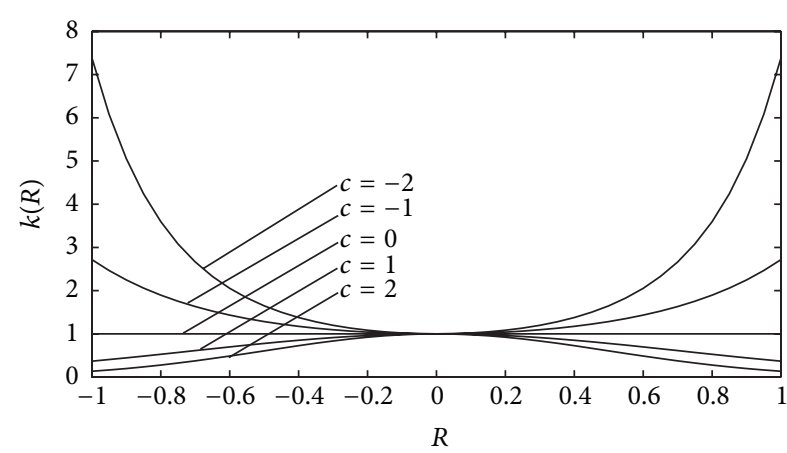

FIgURE 2: Curvature profile.

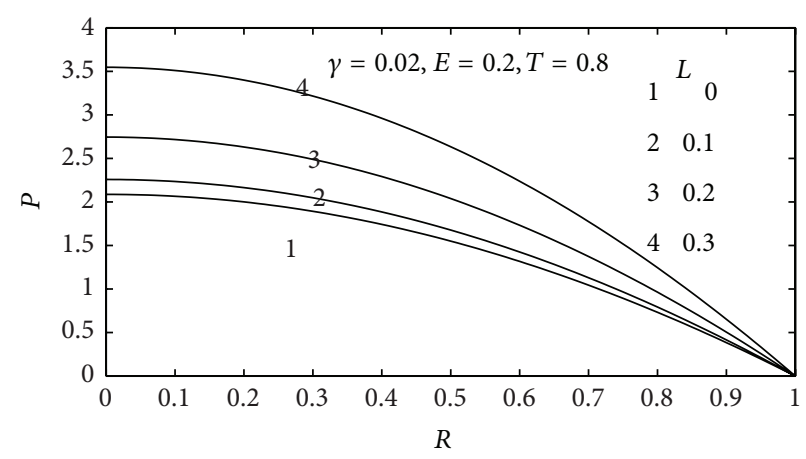

FIGURE 3: Couple stress effects on the squeeze film pressure-flat disk case.

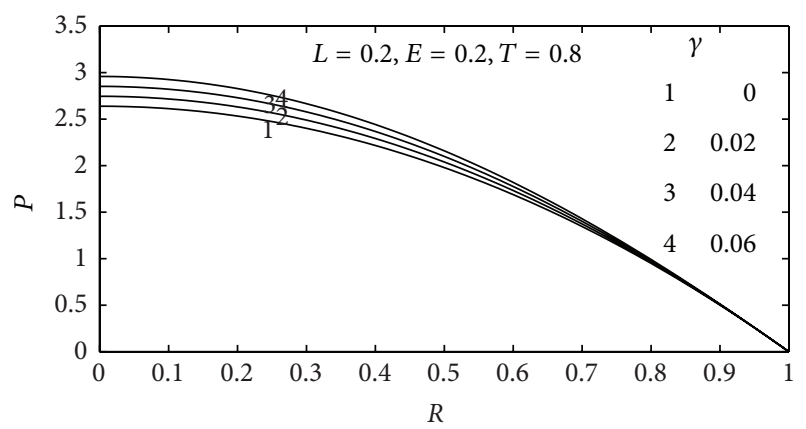

FIGURE 4: Effects of VPD on the squeeze film pressure-flat disk case.

Figures 3-5 present the squeeze film characteristics for the flat disk case. Figure 3 shows the squeeze film pressure $P$ as a function of radial coordinate $R$ for a small amplitude of sinusoidal motion $E=0.2$ at time $T=0.8$ and for the viscosity parameter $\gamma=0.02$ and for various values of the couple stress parameter $L$. Higher pressure is obtained for larger values of $L$ due to the rotations of higher number of microstructures present in the fluid. Figure 4 shows $P$ as a function of $R$ for $E=0.2, T=0.8$, and $L=0.2$ and for different values of $\gamma$. Higher film pressure is obtained by considering $\operatorname{VPD}(\gamma=0.02)$, compared to the isoviscous case $(\gamma=0)$, and an increase in the squeeze film pressure is observed with an increase in the viscosity parameter $\gamma$. 


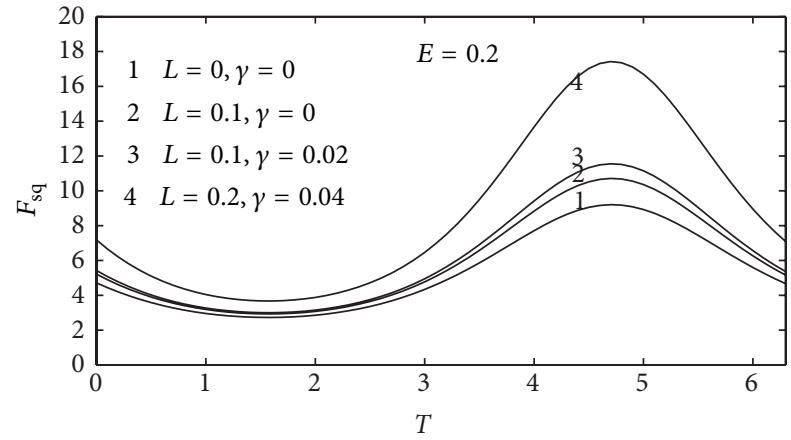

FIGURE 5: Effects of couple stresses and VPD on the squeeze film force-flat disk case.

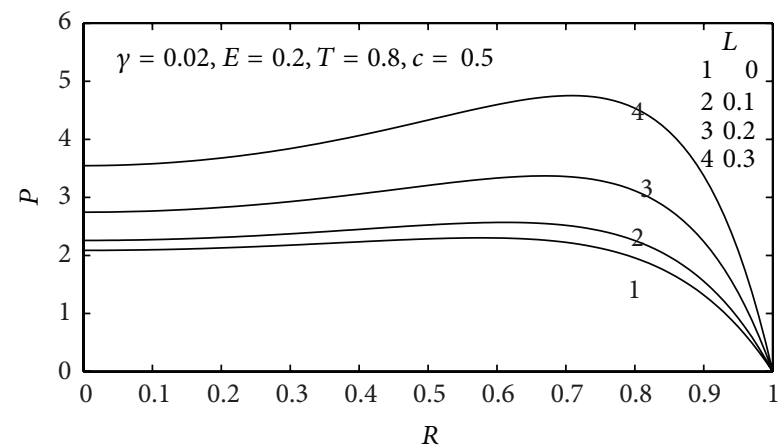

(a)

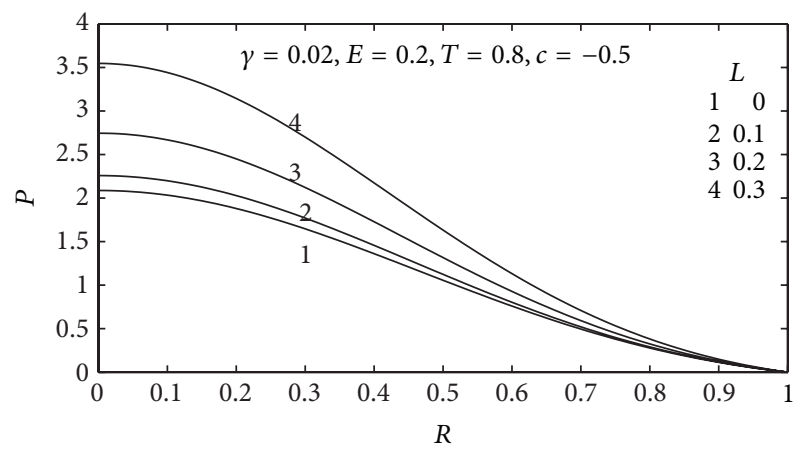

(b)

Figure 6: Couple stress effects on the squeeze film pressure. (a) Concave disk and (b) convex disk.

Figure 5 describes the variation of the load carrying capacity $F_{\mathrm{sq}}$ as a function of response time $T$ for $E=0.2$ and for various values of $L$ and $\gamma$ in the flat disk case. Compared to the isoviscous Newtonian case with $L=0$ and $\gamma=0$, an increase in the load carrying capacity is observed with an increase in the couple stress parameter with $L=0.1$ and $\gamma=0$. Further increase is seen when considering VPD with $\gamma=0.02$ and $L=0.1$. Combined effects of couple stresses and VPD, with $L=0.2$ and $\gamma=0.04$, further enhance the load carrying capacity.

Figures 6-9 present the squeeze film characteristics of the flow in the curved disk case with the same amplitude $E=0.2$ of the sinusoidal motion. Figures $6(\mathrm{a})$ and $6(\mathrm{~b})$

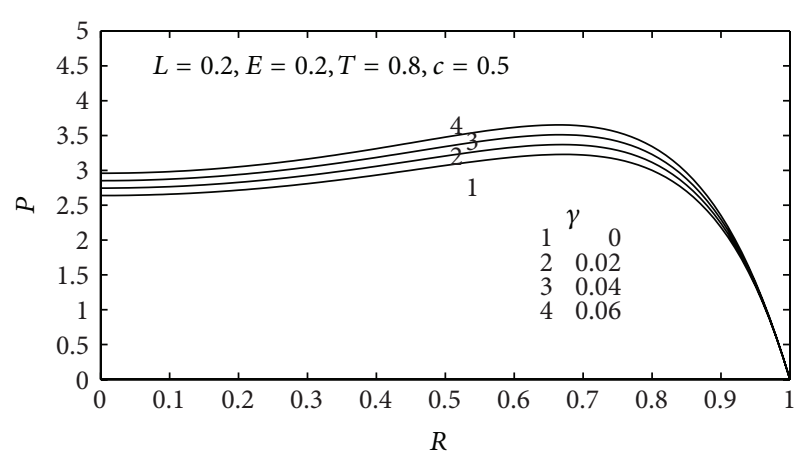

(a)

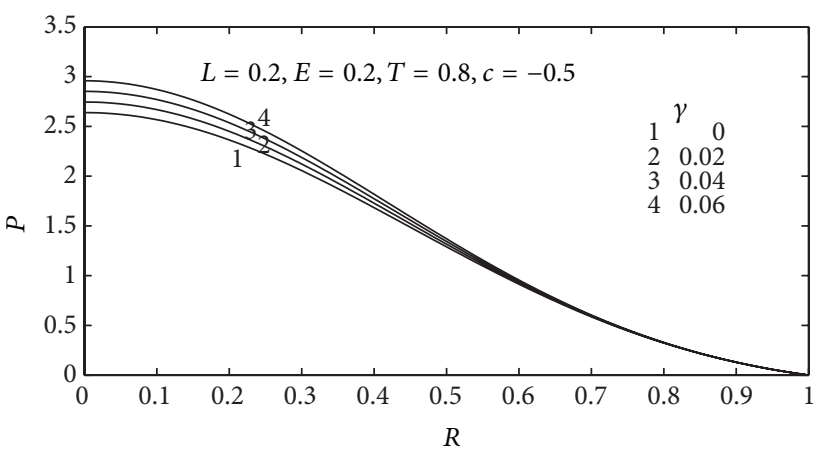

(b)

FIGURE 7: Effects of VPD on the squeeze film pressure. (a) Concave disk and (b) convex disk.

show the pressure graphs for $T=0.8, \gamma=0.02$ and various values of $L$ in the concave $(c>0)$ and convex $(c<0)$ disk cases, respectively. It is observed that in both the cases, the squeeze film pressure increases with an increase in the couple stress parameter $L$. Further, it is seen that the squeeze film pressure is more significant for larger values of $L$ for the concave nature of the upper curved disk. Figures 7(a) and 7(b) display the squeeze film pressure $P$ as a function of radial coordinate $R$ with $T=0.8, L=0.2$ and for various values of $\gamma$ in the concave and convex disk cases, respectively. In both concave and convex disk cases, higher squeeze film pressure is observed by considering VPD with $\gamma=0.02$, compared to the isoviscous case with $\gamma=0$ and a further increase in the squeeze film pressure is observed with an increase in the viscosity parameter $\gamma$, particularly in the concave disk case. Figures 8(a) and 8(b) describe the variation of load carrying capacity $F_{\mathrm{sq}}$ as a function of response time $T$ for $E=0.2$ and various values of $L$ and $\gamma$ in the concave $(c>0)$ and convex $(c<0)$ disk cases, respectively. An increase in the load carrying capacity is obtained with an increase in the couple stress parameter $L$. Further increase in the load carrying capacity is observed when considering VPD for both concave and convex nature of the upper curved disk. Figure 9 shows the variation of load carrying capacity as a function of response time at $L=0.2, \gamma=0.02$ and various values of curvature parameter $c$. It is observed that the load carrying capacity is higher in a concave disk than in a flat disk whereas it is lower in a convex disk than in a flat disk. 


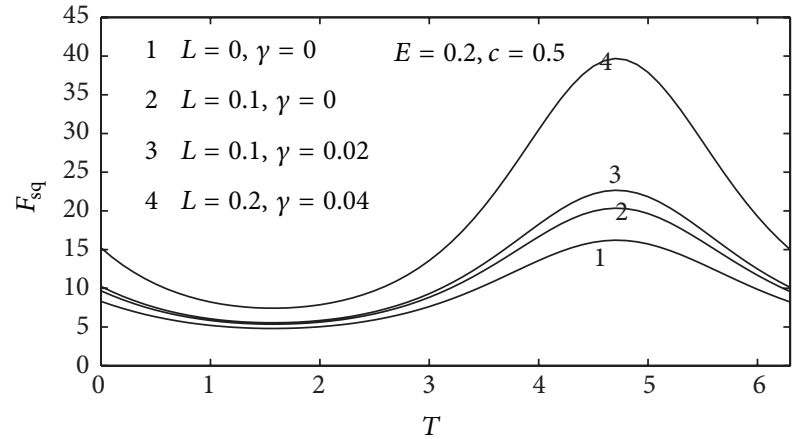

(a)

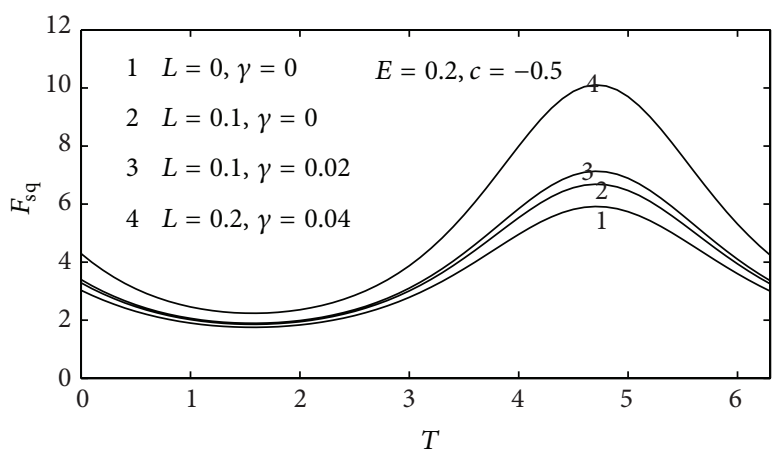

(b)

FIGURE 8: Effects of couple stresses and VPD on the squeeze film force. (a) Concave disk and (b) convex disk.

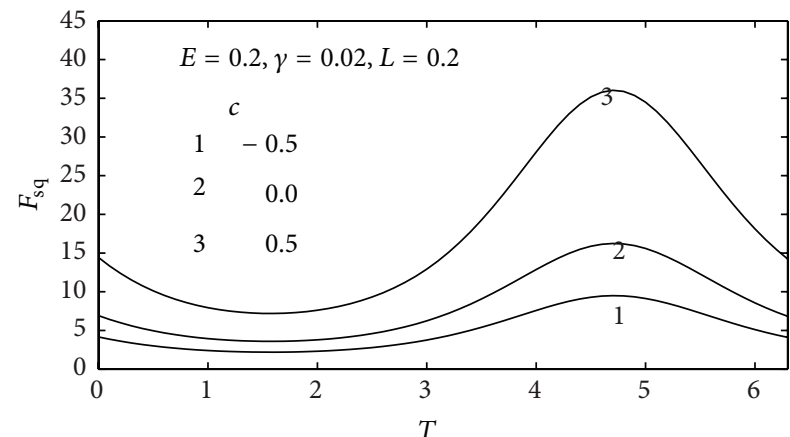

FIGURE 9: Curvature effects on the squeeze film force.

\section{Conclusion}

The combined effects of couple stresses and VPD on the curved squeeze film behaviour are studied through the squeeze film pressure and load carrying capacity. Using Stokes microcontinuum theory and the Barus formula, the Reynolds type equation is derived. The expressions for squeeze film pressure and load carrying capacity are derived using a perturbation technique. It is observed that the effects of couple stresses, variation of viscosity with pressure, and curvature and their combined effects strongly influence the squeeze film behaviour. That is, the larger the value of the couple stress parameter, the greater the load carrying capacity in the squeeze film. This effect is more significant when the viscosity pressure dependence (VPD) is considered. The magnitude of the squeeze film pressure and load carrying capacity on a concave disk is always more than that on a flat disk whereas it is always less for a convex disk which suggests that the load carrying capacity in the squeeze film is strongly influenced by the curvature of the upper moving disk in addition to VPD and couple stress parameters. On the whole the combined effects of curvature VPD and couple stresses on the squeeze film pressure and load carrying capacity are seen to be more significant in a concave disk. Thus, a concave disk is more suitable for practical applications of squeeze film flows with couple stress fluids.

\section{Conflict of Interests}

The authors declare that there is no conflict of interests regarding the publication of this paper.

\section{Acknowledgments}

The authors express their sincere gratitude to the reviewers and the editors for their valuable suggestions to improve the paper in its present form.

\section{References}

[1] O. Pinkus and B. Sternlicht, Theory of Hydrodynamic Lubrication, Mc-Graw Hill, New York, NY, USA, 1961.

[2] A. F. Jones and S. D. R. Wilson, "On the failure of lubrication theory in squeezing flows," ASME Transactions Journal of Lubrication Technology, vol. 97, no. 1, pp. 101-104, 1975.

[3] H. A. Spikes, "The behaviour of lubricants in contacts: current understanding and future possibilities," Proceedings of the Institution of Mechanical Engineers, Part J: Journal of Engineering Tribology, vol. 208, no. 1, pp. 3-15, 1994.

[4] T. T. Ariman, M. A. Turk, and N. D. Sylvester, "Microcontinuum fluid mechanics-a review," International Journal of Engineering Science, vol. 11, no. 8, pp. 905-930, 1973.

[5] T. T. Ariman and N. D. Sylvester, "Applications of microcontinuum fluid mechanics," International Journal of Engineering Science, vol. 2, pp. 273-293, 1974.

[6] V. K. Stokes, "Couple stresses in fluids," Physics of Fluids, vol. 9, no. 9, pp. 1709-1715, 1966.

[7] G. Ramanaiah, "Squeeze films between finite plates lubricated by fluids with couple stress," Wear, vol. 54, no. 2, pp. 315-320, 1979.

[8] N. M. Bujurke and N. B. Naduvinamani, "The lubrication of lightly loaded cylinders in combined rolling, sliding and normal motion with couple stress fluid," International Journal of Mechanical Sciences, vol. 32, no. 12, pp. 969-979, 1990.

[9] S. F. Alyaqout and A. A. Elsharkawy, "Optimal film shape for two-dimensional slider bearings lubricated with couple stress fluids," Tribology International, vol. 44, no. 3, pp. 336-342, 2011.

[10] J.-R. Lin and C.-R. Hung, "Combined effects of non-Newtonian rheology and fluid inertia forces on the non-linear transient behaviour in circular squeeze films," Proceedings of the IMech 
E, Part J: Journal of Engineering Tribology, vol. 221, no. 4, pp. 535-541, 2007.

[11] J.-R. Lin, "Squeeze film characteristics between a sphere and a flat plate: couple stress fluid model," Computers \& Structures, vol. 75, no. 1, pp. 73-80, 2000.

[12] N. B. Naduvinamani, P. S. Hiremath, and G. Gurubasuvaraj, "Effect of surface roughness on characteristics of couplestress squeeze film between anisotropic porous rectangular plates," Fluid Dynamics Research, vol. 32, no. 5, pp. 217-231, 2003.

[13] R. S. Gupta and V. K. Kapur, "The simultaneous effects of thermal and inertia in curved circular squeeze films," ASME Transactions Journal of Lubrication Technology, vol. 102, no. 4, pp. 501-504, 1980.

[14] R. Usha and P. Vimala, "Curved squeeze film with inertial effects-energy integral approach," Fluid Dynamics Research, vol. 30, no. 3, pp. 139-153, 2002.

[15] R. Usha and P. Vimala, "Theoretical investigation of unsteady squeeze flow in a curved Newtonian squeeze film," Journal of Tribology, vol. 124, no. 4, pp. 865-869, 2002.

[16] L. H. Butler, "The hydrodynamic effect between approaching surfaces with interposed viscous fluid films and its influence on surface deformations," Journal of the Institute of Petroleum, vol. 46, pp. 63-65, 1960.

[17] P. Gould, "Parallel surface squeeze film: the effect of the variation of viscosity with temparature and pressure," Journal of Lubrication Technology, vol. 66, pp. 375-380, 1967.

[18] A. Kalogirou, S. Poyiadji, and G. C. Georgiou, "Incompressible Poiseuille flows of Newtonian liquids with a pressuredependent viscosity," Journal of Non-Newtonian Fluid Mechanics, vol. 166, no. 7-8, pp. 413-419, 2011.

[19] R. F. Lu and J. R. Lin, "A theoretical study of combined effects of non-Newtonian rheology and viscosity-pressure dependence in the sphere-plate squeeze-film system," Tribology International, vol. 40, no. 1, pp. 125-131, 2007. 

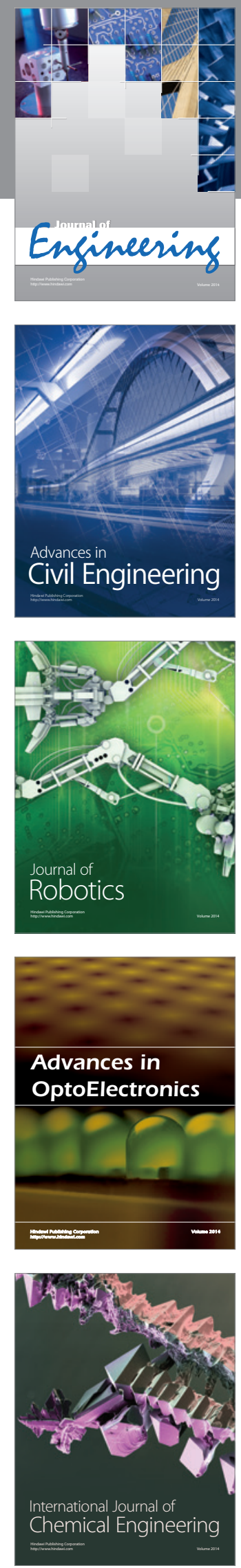

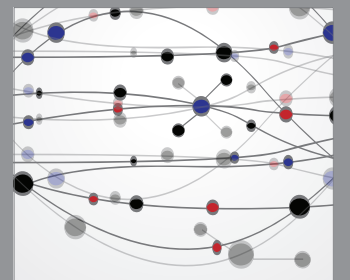

The Scientific World Journal
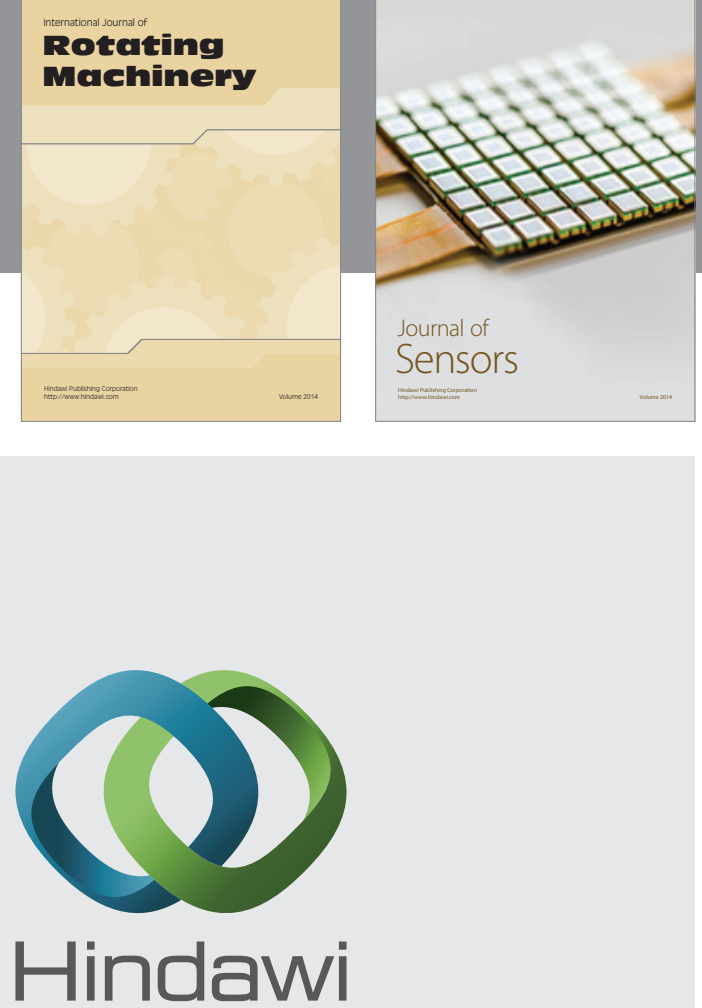

Submit your manuscripts at http://www.hindawi.com
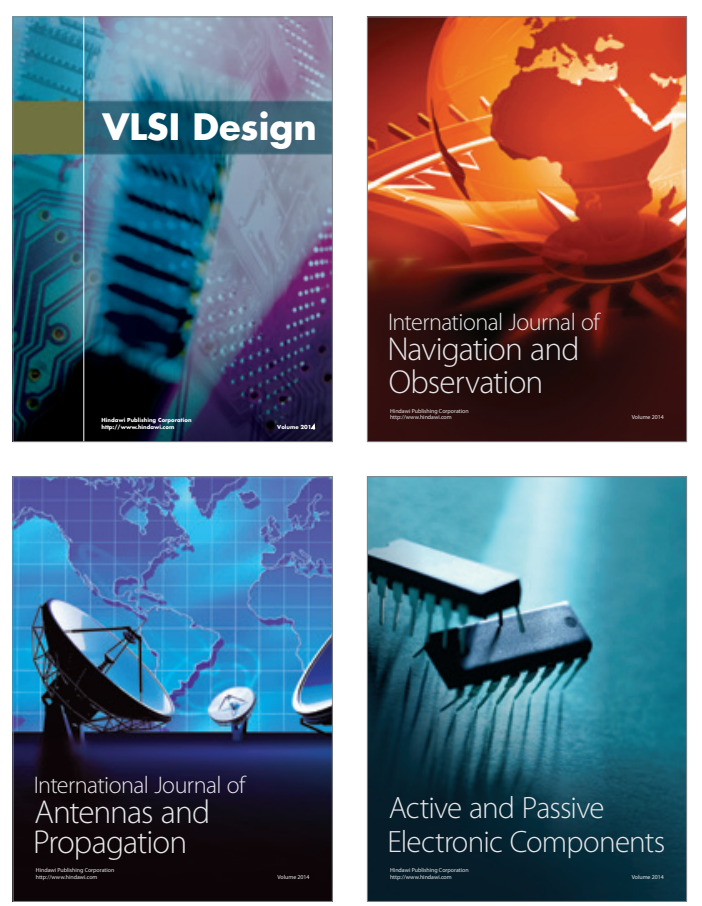
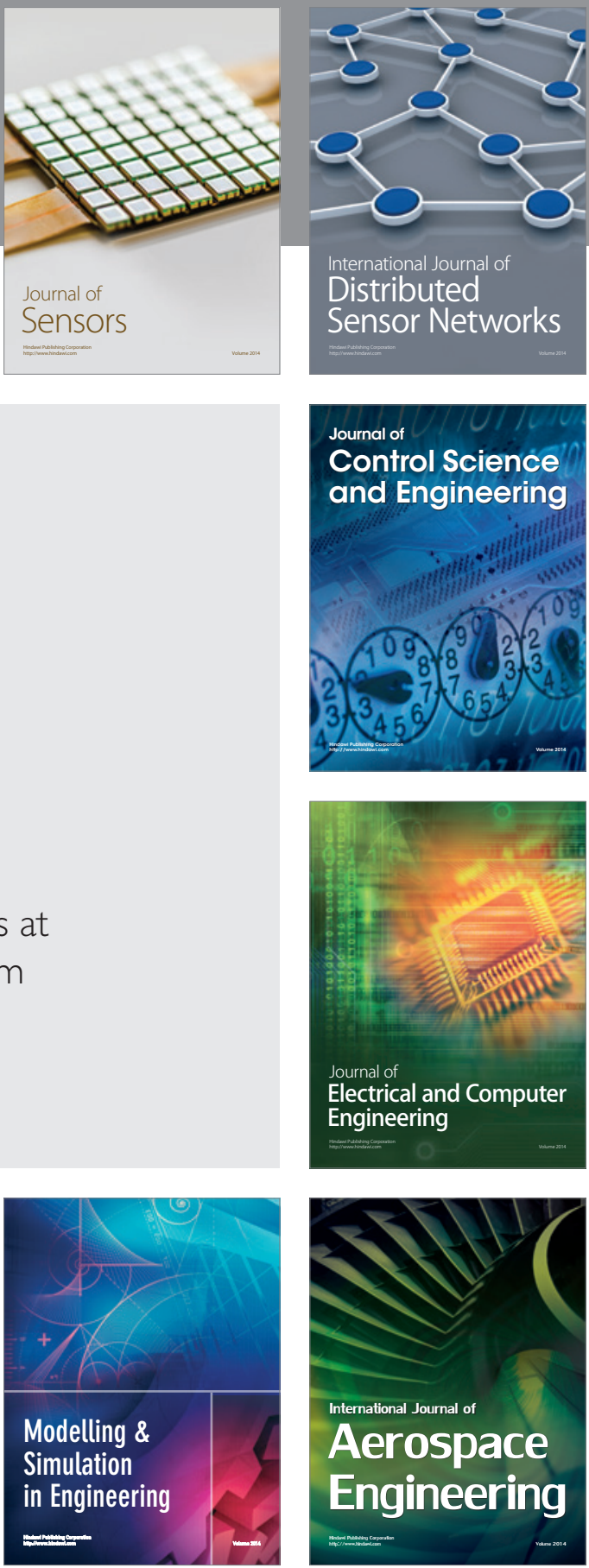

Journal of

Control Science

and Engineering
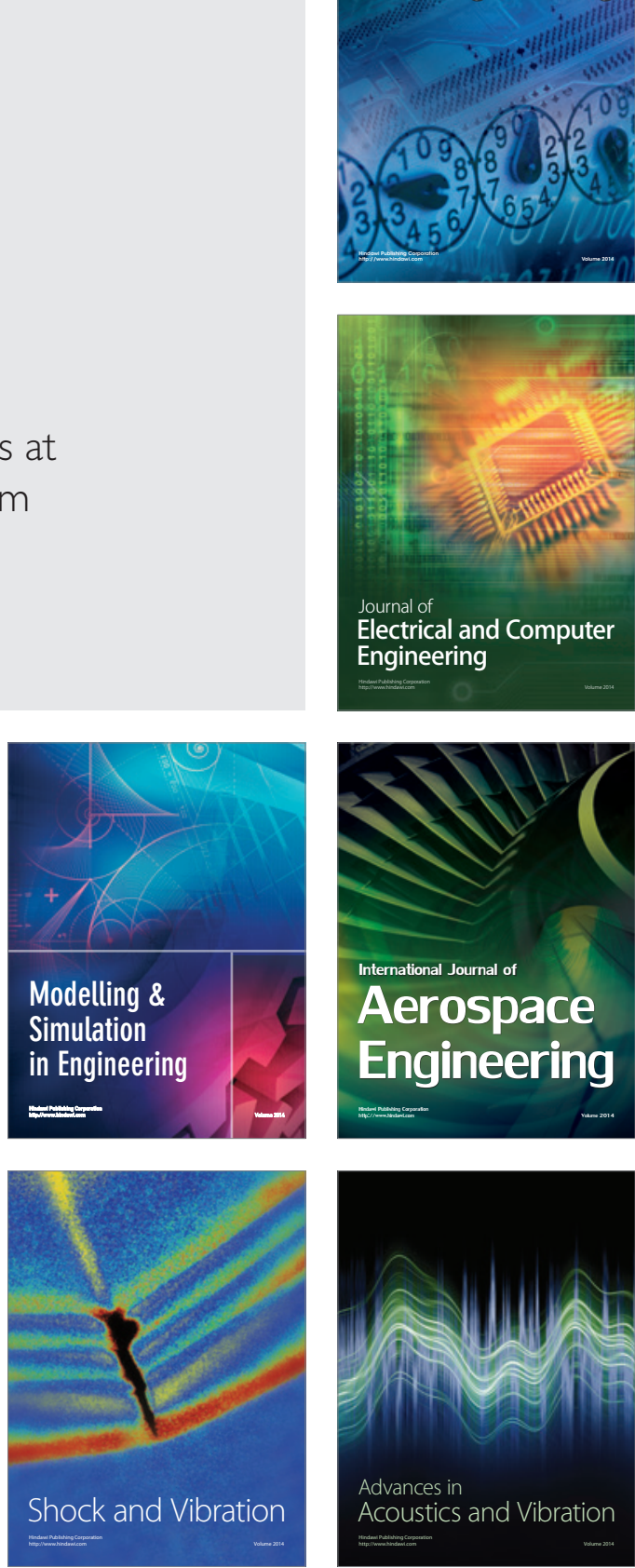